\title{
Studies of some genes of Bacillus subtilis MN99 local strains against plant diseases
}

\author{
Ariunaa Saraadanbazar', Byambasuren Mijidsuren, Battur Banzragch²:(D) \\ ${ }^{1}$ Laboratory of Microbiology, Institute of Plant Protection, Mongolian University of Life Science, Zaisan 17024, \\ Ulaanbaatar, Mongolia \\ ${ }^{2}$ Graduate school, Mongolian University of Life Sciences, Zaisan 17024, Ulaanbaatar, Mongolia
}

*Corresponding author: battur.b@muls.edu.mn

https://orcid.org/0000-0002-2573-8031

\begin{tabular}{lll}
\hline Received: 30.03 .2021 & Revised: 13.09 .2021 & Accepted: 15.09 .2021 \\
\hline
\end{tabular}

\begin{abstract}
The objective of this study was the isolation and characterization of Bacillus subtilis local strains from the soil in Mongolia. These local strains of $B$. subtilis are showed to have high antagonistic activities against some plant pathogenic fungi and bacteria. Six strains of B. subtilis were isolated and characterized morphologically, physiologically and biochemically according the Bergey's Manual of Systematic Bacteriology.

In order to identify species of the isolated strains, we amplified and sequenced $16 S r R N A$ gene, essential funtinal genes bmyB, spoVG and $s r f A$, which are related to antagonistic activity of these strains. The sequences were aligned using CLASTALW multiple sequence alignment tool. Phylogenetic tree was drawn according to Maximum likelihood"method and "Tamura-Nei" model using "MEGA-X version 10.2.6 program.

Among all isolates of $B$. subtilis MN99 and 7/24 strains had higher antagonist activity against plant diseases. According to partial sequence of srfAA (620bp) gene of MN99, the local strain belongs to B. subtilis and partial sequence of bmyB (370bp), spoVG (22bp) gene of MN7/24 strain showed that the it belongs to B. atrophaeus species. All local strains of B. subtilis had bacillomycin synthesis gene, and B. subtilis MN99 strain had only surfactine synthesis gene, while did not have spore formation and hemolysis gene SpoVG.
\end{abstract}

Keywords: Local strain, antimicrobe peptide gene, biocontrol

\section{Introduction}

Application of microorganisms can provide environmentally-friendly solution to secure sustainable agricultural and food production. For this reason, several bacterial strains, including Bacillus, Pseudomonas, and Streptomyces have been practiced in agriculture. Particularly, Bacillus species are considered as an effective due to their resistance to adverse environmental conditions and high ability to control against broad range of plant pathogens [1]. Also, they have an ability to produce various antimicrobial peptides and compounds, such as volatile organic compounds, protein and enzymes. Additionally, this strains can vary both phenotypically and genetically for their antagonistic potentiality [2].

Any strain of $B$. subtilis group is estimated that at least $4-5 \%$ of its genome is responsible for the production of antimicrobial compounds (AMCs) production. These molecules are mainly antimicrobial peptides (AMPs) [3]. Recently, use of 16S rRNA gene sequences in phylogenetical and taxanomic studies of bacteria became the most common technique for several reasons: (i) it is presented in almost all bacteria, often existing as a multigene family, or operons; (ii) the function of the $16 S$ rRNA gene is stable, thereby, random sequence changes are measurable for time in terms of evolution; and (iii) size of the $16 \mathrm{~S}$ rRNA gene $(1,500 \mathrm{bp})$ is enough for informatical purpose [4].

Especially, powerful antifungal metabolites (lipopeptides of the surfactin, iturin and fengycin families) produced by strains of $B$. subtilis, are important for plant protection purposes. Because of its unique biochemical characteristics, antibiotic 
production by these strains plays a major role in plant disease suppression [5]. As a result of this study, six strains of $B$. subtilis were isolated from local soil, its $16 S$ rRNA was amplified, and important functional

\section{Materials and methods}

\section{DNA extraction from Bacillus subtilis and PCR}

Six isolates of $B$. subtilis have been cultured and maintained on Nutrient agar (NA) plate at $35^{\circ} \mathrm{C}$. In order to extract DNA from pure culture of bacteria, small portion of bacterial mass on NA was collected by loop and added in $450 \mu \mathrm{L}$ of DNA lysate buffer in $1.5 \mathrm{ml}$ Eppendorf tube. After mixing this lysate mixture, $5 \mu \mathrm{L}$ of proteinase $\mathrm{K}$ mixture was added, and incubated for 6-12 hour at $55 \mathrm{C}^{\circ}$ to lyse bacterial cells. Then, $500 \mathrm{~mL}$ of phenol: chloroform: isoamyl alcohol mixture was added to the lysate, and mixed by vortex for 1 minute. The vortexed mixture was centrifuged at $15000 \mathrm{rpm}$ for $30 \mathrm{~min}$. After the centrifugation, supernatant containing DNA transferred to new tube with $400 \mathrm{ml}$ of phenol: chloroform: isoamyl alcohol to the supernatant, the mixed by vortex for 1 minute. The vortexed mixture was centrifuged at $15000 \mathrm{rpm}$ for 10 minute. Then, supernatant was gently pipetting out. Pellets were added with $50 \mu 13 \mathrm{M} \mathrm{NaCI}$ and $1 \mathrm{~mL} 99 \%$ of cool ethanol and incubated at $-20 \mathrm{C}^{\circ}$ for 30 minute. and specific genes, such as bmyB, spoVG and srfAA and phylogenetic relationships were analyzed using parts of amplified gene sequences.
The supernatant was discarded after centrifugation at $15000 \mathrm{rpm}$ for $30 \mathrm{~min}, 100 \mathrm{~mL}$ of $70 \%$ ethanol was added to the pellet, and then mixture was centrifuged at $15000 \mathrm{rpm}$ for 15 minute. Supernatant was discarded and pellet in the tube was left for drying on air around 20 minute in order to completely evaporate residual alcohol. After this step, the pellets were resuspended in $100 \mathrm{~mL}$ of deionized and distilled water. After the pellet dissolve in the water, we measured the purity and concentration of the extracted DNA by spectrophotometer. The DNA was stored and maintained at $-20 \mathrm{C}^{\circ}$ to $-40 \mathrm{C}^{\circ}$. Initial denaturation at $95 \mathrm{C}^{\circ}$ for 5 minute, followed by 35 cycle of denaturation at $95 \mathrm{C}^{\circ}$ for 1 minute, annealing at $59(60) \mathrm{C}^{\mathrm{o}}$ for 1 minute and extension at $72 \mathrm{C}^{\circ}$ for 50 second. Then final extension at $72 \mathrm{C}^{\circ}$ for 7 minutes was performed and after the final extension temperature dropped to $4 \mathrm{C}^{\mathrm{o}}$ until the remove from thermocycler. Primer that used in this study was shown in table 1 .

Table 1

Used primer list

\begin{tabular}{|c|c|c|c|c|}
\hline Target & Gen & Primer name & Primer sequence & bp \\
\hline \multirow{2}{*}{$16 \mathrm{~S}$ rRNA } & \multirow{2}{*}{ 16SrDNA } & $16 \mathrm{SbacF}$ & GCTTGCTCCCTGATGTTAGC & \multirow{2}{*}{163} \\
\hline & & 16SbacR & CGGGTCCATCTGTAAGTGGT & \\
\hline \multirow{2}{*}{ Spore protein } & \multirow{2}{*}{ spoVG } & spoF & AATACCGATGGTCGCATGA & \multirow{2}{*}{226} \\
\hline & & spoR & CAGAATCACCCAAACGATGA & \\
\hline \multirow{2}{*}{ Bacyllomicin } & \multirow{2}{*}{ bmyB } & bmyBF & GAATCCCGTTGTTCTCCAAA & \multirow{2}{*}{370} \\
\hline & & bmyBR & GCGGGTATTGAATGCTTGTT & \\
\hline \multirow{2}{*}{ Surfactin } & \multirow{2}{*}{ srfAA } & srfaF & TCGGGACAGGAAGACATCAT & \multirow{2}{*}{620} \\
\hline & & srfaR & CCACTCAAACGGATAATCCTGA & \\
\hline
\end{tabular}

Determination of biological activity encoding some genes of B. subtilis

PCR was performed in order to detect types of $B$. subtilis strains using primer that specifically designed for amplification of $16 S R N A$ gene fart. Furtherly, bmyB, spoVG, and srfAA genes that encode active biological proteins such as bacyllomycine, spore protein and surfactin. We have sequenced that amplified product and compared other similar sequence in gene bank.

\section{Results and discussion}

Phylogenetic analysis and sequencing of the $16 \mathrm{~S}$ ribosomal RNA gene of B. subtilisBacillus genus
Because we have found similar sequences from NCBI data base using BLAST web tool, twenty of these similar sequences were chosen to compare. Selected twenty sequences were aligned using CLASTALW multiple sequence alignment tool. Phylogenetic tree was drawn according to "Maximum likelihood" method and TamuraNei"model using MEGA-X version 10.2.6"program [6].

bacteria and their species classification and subtyping were performed using $16 S$ ribosomal $R N A$ gene, 
which is common molecular genetics methods. DNA extraction from $B$. subtilis local isolate was done with generic DNA extraction method as mentioned previously. PCR was performed using primer which is specifically designed for amplification of $16 S R N A$ gene fart. Results are shown in figure 1.

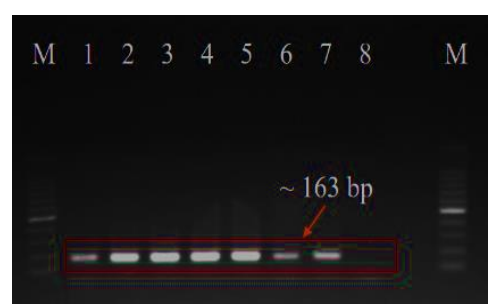

Figure 1. Results of amplification of 16S ribosomal RNA gene

M-100 bp step DNA size marker, 1-B. subtilis ATCC 6633 strain, 2-B. subtilis local strain MN1, 3- B. subtilis local strain MN300, 4- B. subtilis local strain 70, 5- B. subtilis local strain MN7/24, 6- B. subtilis local strain Y7, 7- B. subtilis local strain MN99, 8-Negative control

According to Figure 1, standard strain on line 1 and other local strain on line 2-7 were all shown same

Determination of some genes of $B$. subtilis that are responsible for biological activities

bmyB, spoVG, srfAA genes of B. subtilis of local strains that encodes synthesis of antibiotic-like chemicals such as bacyllomycin, spore protein, surpactine determined by PCR. band with length of $163 \mathrm{bp}$. This indicated that new isolates were all belonged to $B$. subtilis.

DNA extraction PCR amplification was performed as mentioned previously. PCR result was shown as follow as line 1 was $B$. subtilis ATCC 6633 strain, from line 2 to line 7 are local strains of $B$. subtilis. Line 8 is negative control. Research results were shown in Figure 2.

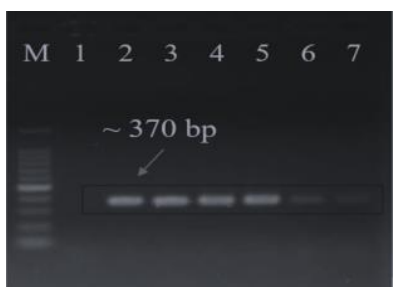

Figure 2. Results of PCR amplifying bmyB (370bp) gene

M-100bp size marker 1-B. subtilis ATCC 6633 strain, 2-B. subtilis local strain MN1, 3- B. subtilis local strain MN300, 4- B. subtilis local strain 70, 5- B. subtilis local strain MN7/24, 6- B. subtilis local strain Y7, 7- B. subtilis local strain MN99, 8-Negative control

As shown in Figure 2, B. subtilis ATCC 6633 strain did not showed the band. On contrary, local strains (on line 2-7) showed band with length of $370 \mathrm{bp}$. This result indicated this strain possessed $b m y B$ gene which is responsible for synthesis of Baccyllomycin. Above mentioned, 6 strains were further subjected to DNA sequences of bmyB gene. B. subtilis strain 70 and $B$. subtilis MN7/24 were successfully sequenced about 222-264 bp in length. Sequences of bmyB genes of these two strains were same and showed $100 \%$ of similarity. Based on $b m y B$ gene sequences, phylogenetic tree showed these strains have some similarity with Bacillus atrophaeus. 


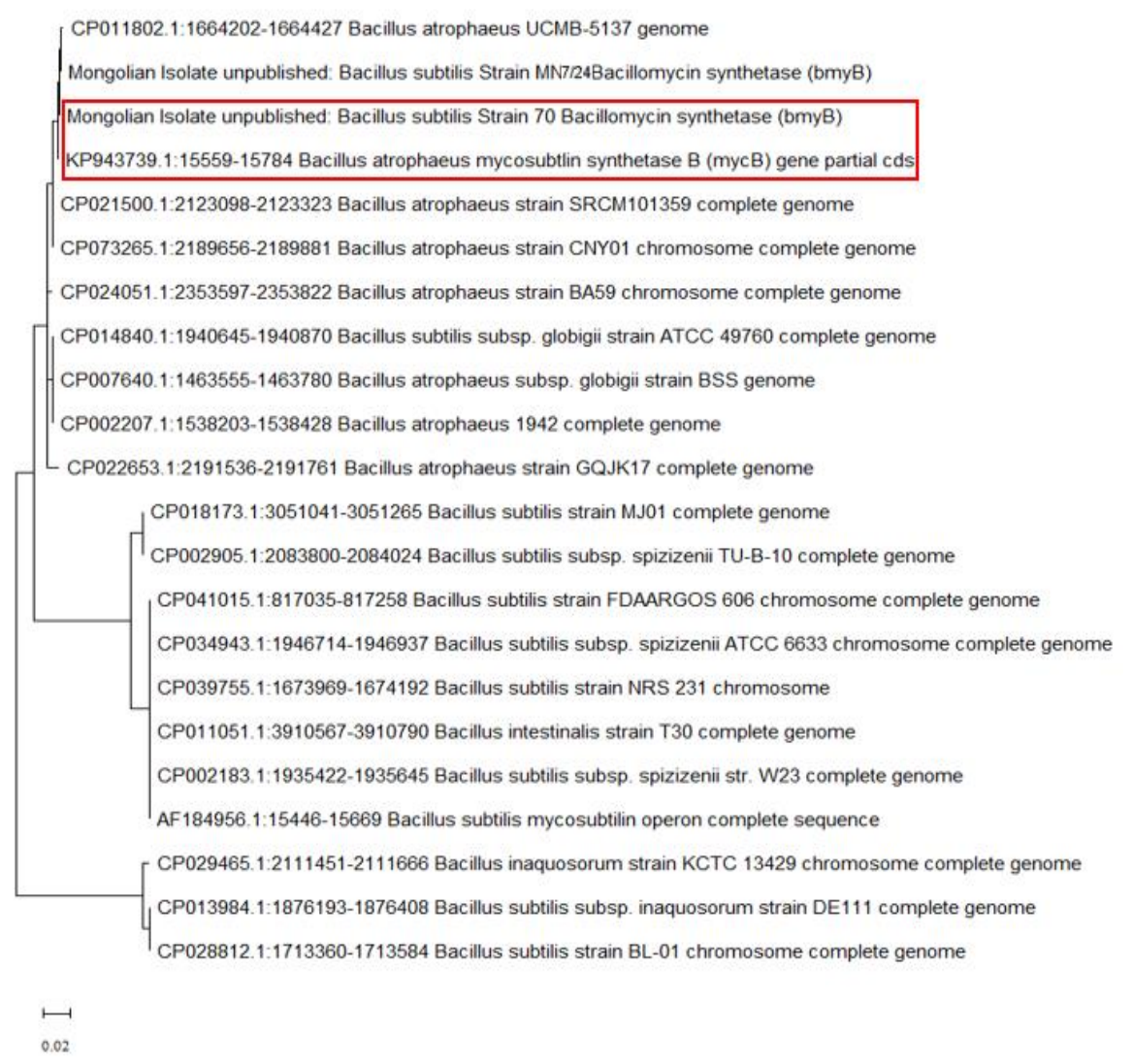

Figure 3. Phylogenetic relationships of Bacyllomycin bmyB gene of local B. subtilis MN 7/24 and 70 strains based phylogenetic tree

Results based on bmyB gene sequences, phylogenetic tree showed that B. subtilis MN7/24 and 70 isolates are grouped to one groups that is

Determination of spoVG gene that is responsible for hemolysis and spore formation

The local strain of $B$. subtilis and standard strain ATCC 6633 was examined for presence of a $s p o V G$

subtypes of Bacillus atrophaeus and B. subtilis subsp. globigi.

gene which is responsible for hemolysis and spore forming. In order to determine spoVG gene, PCR was performed and the results was shown in Figure 4.

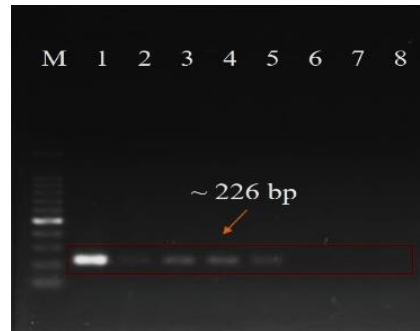

Figure 4. PCR result of spoVG gene amplification M- DNA size marker with 100 bp step

1-B. subtilis ATCC 6633 strain, 2-B. subtilis local strain MN1, 3- B. subtilis local strain MN300, 4- B. subtilis local strain 70, 5- B. subtilis local strain MN7/24, 6- B. subtilis local strain Y7, 7- B. subtilis local strain MN99, 8-Negative control

Based on Figure 4, Spore formation spoVG gene are present in both $B$. subtilis ATCC 6633 strain and local strains on line 2-5. As represented in the figure, B. subtilis ATCC and local strains on the line 2-7 are amplified 226 bp PCR amplicon. However local strain MN99 and B. subtilis Y7 strains are absence of this gene. We have purified this PCR amplicon and sequenced this two short sequences. Based on these two sequences, we constructed phylogenetic tree and result was shown in Figure 5. 

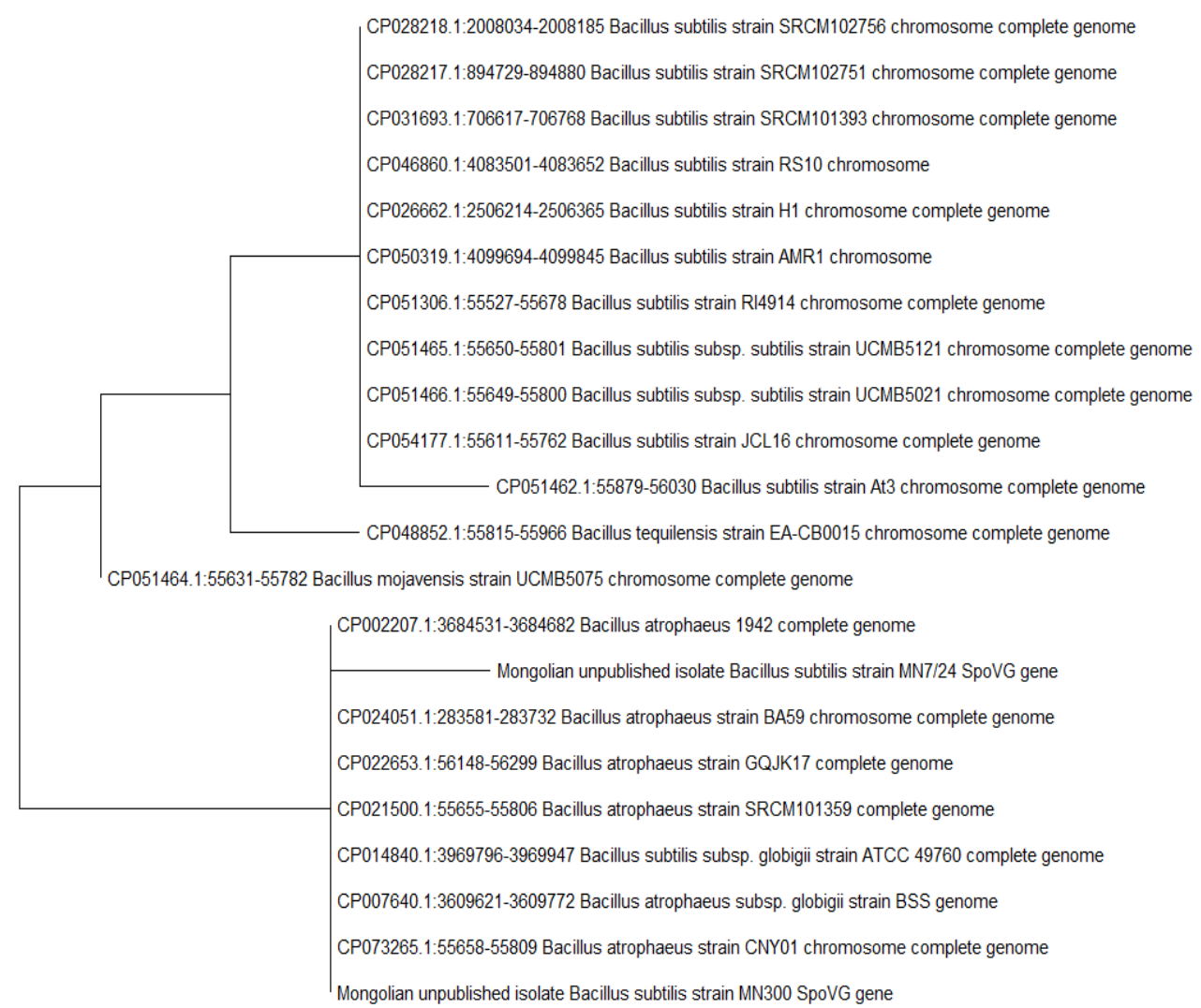

0.0050

Figure 5. Phylogenetic tree based spoVG gene of B. subtilis strain MN7/24

This results indicated that $B$. subtilis MN7/24 strain is similar to B. atrophaesus subtype in terms of spoVG gene.

Detection of Surfactin synthesis srfAA gene Lipopeptides are surfactants of microorganisms that are widely used in the biotechnological production of drugs and preparation against human, animal and plant diseases. Bacillus genus of bacteria is the producers of these active compounds, including $B$. subtilis, which produces surfactin, the strongest biosurfactant known nowadays.

Surfactin, secretes by $B$. subtilis, is antibiotic-like, antiviral, anti-cancer compound which functions as immunomodulatory. Therefore, we used PCR to determine whether local strains contain genes that produce surfactin.

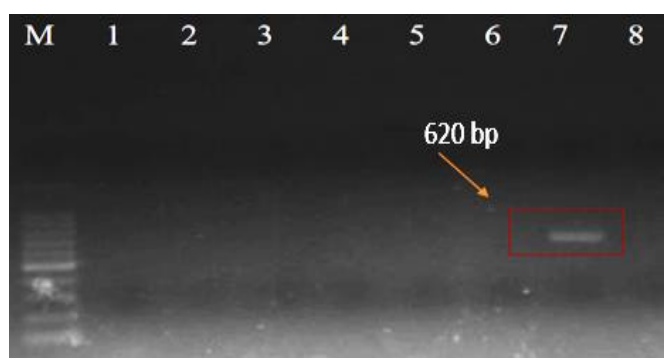

Figure 6. PCR result of amplification of srfAA (620 bp) gene

Line M-100 bp step DNA marker, 1-B. subtilis ATCC 6633 strain, 2-B. subtilis local strain MN1, 3- B. subtilis local strain MN300, 4- B. subtilis local strain 70, 5- B. subtilis local strain MN7/24, 6- B. subtilis local strain Y7, 7- B. subtilis local strain MN99, 8-Negative control

According to our findings, B. subtilis ATCC 6633 strain and B. subtilis local strain 2-6 did'nt show presence of surfactin gene srfAA. MN 99 (line) showed presence of surfactin gene srfAA $(620 \mathrm{bp}$ band on gel). This suggests that local strains of $B$. subtilis MN99 might be more biologically active than other strains. 


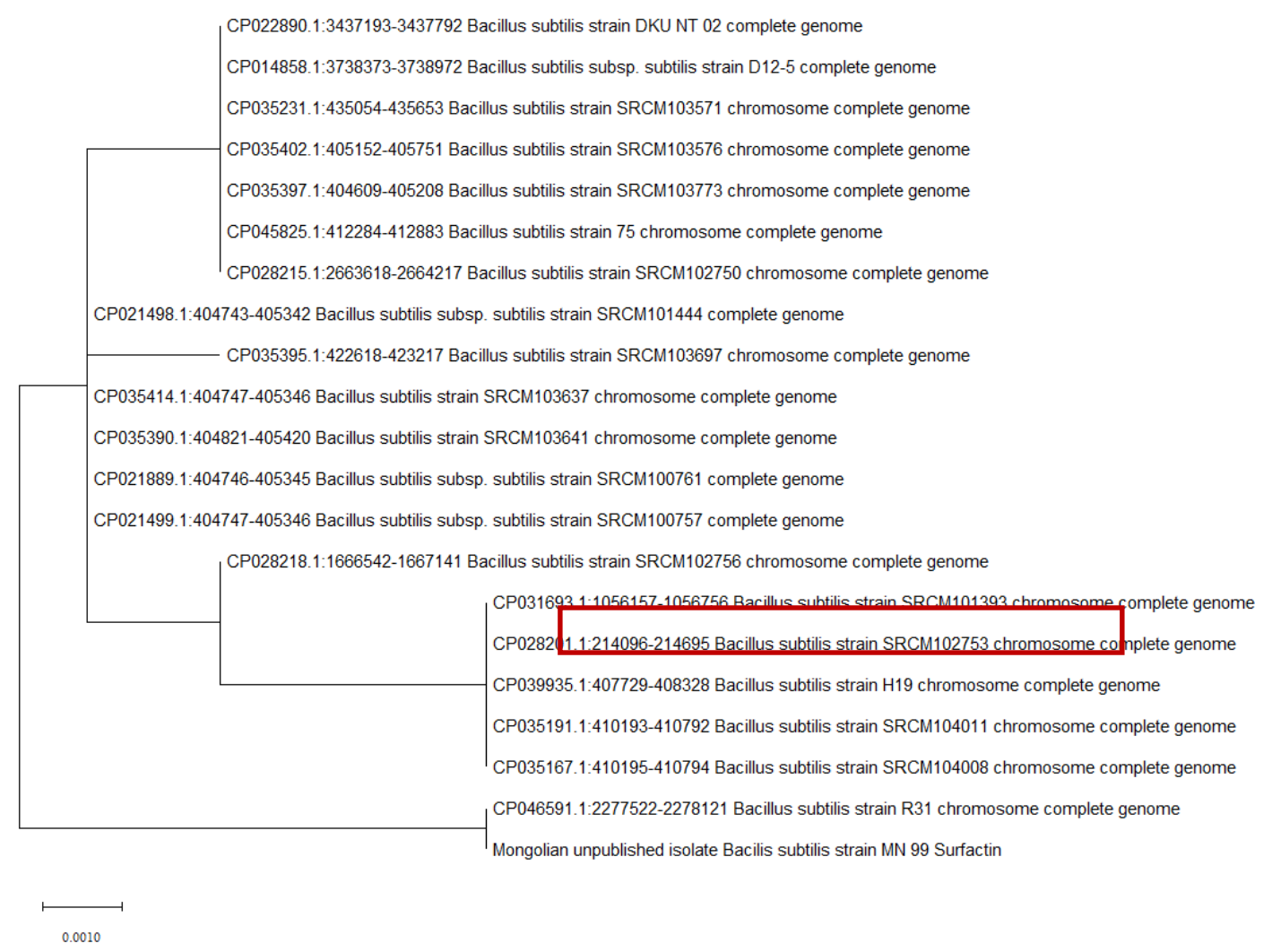

Figure 7. Phylogenetic tree based on srfAA gene of B. subtilis local strain MN99

According to the result, B. subtilis MN99 local strain showed the presence of surfactin gene $\operatorname{srfAA}(620 \mathrm{bp}$ band on gel). Phylogenetic tree indicated that this

\section{Conclusion}

According to our study objective, we have successfully isolated and characterized local strains of $B$. subtilis which has antagonist activity against plant pathogens such as fungi and bacteria. This species of strain is known to yield broad range of bioactive compound, therefore, it is one of the key and powerful biomaterial for preparation of biocontrol agents against plant diseases without harmful effect on environment.

Besides, taxomic identification of isolates, we have determined phylogenetic relationships of strains based on presence of important functional genes responsible for synthesis of some biological active compounds. PCR using the general primers for Bacillus is that based on the 16S rRNA and spoVG, srfA $A$ and $b m y B$ genes resulted in amplifications for the six Bacillus strains that we tested. For functionality of these gene, although spoVG was determined in 1977, the function of this gene has not been clarified for years. This gene is considered to be involved in spore formation and hemolysis [6]. Additionally, srfAA gene which is responsible for strain has close relation with $B$. subtilis in terms of srfAA gene.

synthesis of the small molecule surfactin has been tested for its presence in strains. Surfactin is a powerful surfactant that has been implicated in permitting cell motility over surfaces. Also, surfactin has antifungal and antibacterial properties in vitro that makes it an important natural compound in areas of agriculture [7]. Also presence of $b m y B$ that is responsible for synthesis of bacyllomicin indicates biocontrol ability of strains. Finding of this study can be concluded as follows:

1. Comparative studies of molecular genetic of the partial sequences of $16 \mathrm{~S}$ ribosomal RNA gene suggested that the MN99 local strain was Bacillus subtilis and the MN7/24 local strain was Bacillus atrophaeus.

2. All local strains of $B$. subtilis showed presence of the bacillomycin-synthesis gene $b m y B$, while the $B$. subtilis MN99 local strain had only the surfactin-producing gene surfAA, spoVG gene which involves in sporulation and hemolysis was not found in the strain MN99. 


\section{References}

[1] "Bacillus Species as Biocontrol Agents for Fungal Plant Pathogens," Bacilli and Agrobiotechnology: Phytostimulation and Biocontrol, vol. 2, pp. 239-265, 2019.

[2] Ayslu Mirkasimovna Mardanova, Guzel Fanisovna Hadieva, Marat Tafkilevich Lutfullin, Irina Valer'evna Khilyas, Leyla Farvazovna Minnullina, Adelya Gadelevna Gilyazeva, Lidiya Mikhailovna Bogomolnaya, Margarita Rashidovna Sharipova, "Bacillus subtilis Strains with Antifungal Activity against the Phytopathogenic Fungi," Agriculture science, vol. 8, no. 1, 2017.

[3] Simon Caulier,Catherine Nannan, Annika Gillis, Florent Licciardi, Claude Bragard and Jacques Mahillon, "Overview of the Antimicrobial Compounds Produced by Members of the Bacillus subtilis Group," Front Microbiology, vol. 10, 2019.

[4] J. Michael Janda and Sharon L. Abbott, "16S rRNA Gene Sequencing for Bacterial Identification in the Diagnostic Laboratory: Pluses, Perils, and Pitfalls," American Society for Microbiology Journal of Clinical Microbiology, vol. 45, no. 9, pp. 2761-2764, 2007.
[5] Marc Ongena, Philippe Jacques, Yacine Touré, Jacqueline Destain, Abdelhamid Jabrane, Philippe Thonart, "Involvement of fengycintype lipopeptides in the multifaceted biocontrol potential of Bacillus subtilis," Applied Microbiology and Biotechnology, vol. 69, pp. 29-38, 2005.

[6] Altchul S.F, Gish W, Miller W, Myers E.W and Lipman D.J, "Basic local alignment search tool," Journal of Molecul biology, vol. 215, pp. 403-410, 1990.

[7] Thomas P. Burkea, Daniel A. Portnoya, "SpoVG Is a Conserved RNA-Binding Protein That Regulates Listeria monocytogenes Lysozyme Resistance, Virulence, and Swarming Motility," American society for microbiology, vol. 7 , no. 2 e00240-16, 2016.

[8] Heidi A. Arjes, Lam Vo, Caroline M. Dunn, Lisa Willis, Christopher A. DeRosa, Cassandra L. Fraser, Daniel B. Kearns, and Kerwyn Casey Huang, "Biosurfactant-Mediated Membrane Depolarization Maintains Viabilityduring Oxygen Depletion in Bacillus subtilis," Current Biology, vol. 30, p. 10111022, 2020. 